\title{
Investigating Freeway Traffic Hypercongestion between an On-ramp and its Immediate Upstream Off-ramp*
}

\author{
Y.E. Ge ${ }^{a, b, 1}$, Chong-Feng Xu ${ }^{c}$, W.Y. Szeto ${ }^{d}$, B.R. Sun ${ }^{b}$, H.M. Zhang ${ }^{e}$ and L.W. Zhang ${ }^{c}$ \\ ${ }^{a}$ College of Transport $\& 3$ Communications, Shanghai Maritime University, Shanghai 201306, China \\ ${ }^{b}$ School of Transportation \& Logistics, Faculty of Infrastructure Engineering, Dalian University of Technology, \\ Liaoning 116024, China \\ ${ }^{c}$ School of Mathematical Sciences, Dalian University of Technology, Liaoning 116024, China \\ ${ }^{d}$ Department of Civil Engineering, The University of Hong Kong, Pokfulam, China \\ ${ }^{e}$ Institute of Transportation Studies, University of California, Davis, 2028 Academic Surge, One Shields Avenue, \\ Davis, CA 95616, U.S.A.
}

\begin{abstract}
This paper applies the freeway traffic congestion dynamics proposed in Laval and Leclercq (Continuum Approximation for Congestion Dynamics Along Freeway Corridors. Transportation Science, 87-97) but with a modified distribution scheme of freeway merging flows to investigation of traffic hypercongestion on a freeway between an on-ramp and its immediate upstream off-ramp. The reason to make this modification is that the original merging scheme of this dynamics is found to possibly give undesired full priority to the traffic from the on-ramp and also occasionally make the resulting flow going through the merge greater than the available capacity. Traffic hypercongestion in this paper refers to a state where speed and flow change in the same direction as density varies. A homogeneous freeway segment chosen for this investigation includes an on-ramp and an off-ramp, which may correspond to a freeway passing by a city or town, with an off-ramp lying upstream towards the city and an on-ramp downstream. The entry flow from the upstream approach of the freeway was fixed and constant within the time horizon while both deterministic and random on-ramp inflow rates were used in this investigation. Then the formation and dissipation of traffic hypercongestion is investigated as on-ramp demand and off-ramp departure profiles vary. The first finding of this investigation is that the density in the hypercongestion area behind the merge never reached the jam density. Second, the hypercongested area continued to grow as long as the sum of the demand from the upstream of the freeway and that from the on-ramp was greater than the available capacity at the merge. Third, as long as the rate of flow leaving from the freeway via the off-ramp was not smaller than the entry flow rates from the on-ramp, no hypercongestion had been observed. In addition, in analyzing the numerical results, a series of discussions were carried out to build the linkage between the on- and off-ramp flow profiles and the generated and attracted demand of the city plus its adjacent area the two ramps served for; these discussions suggest that the balanced generated and attracted demand of the city plus its adjacent area can reduce or even remove traffic hypercongestion behind the merge on the chosen freeway segment.
\end{abstract}

Keywords Traffic hypercongestion, freeway congestion dynamics, kinematic wave model of traffic flow, merge

\footnotetext{
*Published in Transportmetrica A: Transport Science at http://dx.doi.org/10.1080/23249935.2014.945509, February 2015

${ }^{1}$ Corresponding author.

College of Transport \& Communications, Shanghai Maritime University, 1550 Haigang Avenue, Lin-Gang, Pudong, Shanghai 201306, China; Email: yege@shmtut.edu.cn; Phone: +86(0)21 38282301
} 


\section{Introduction}

Traffic hypercongestion ${ }^{2}$, where both speed and flow decrease as the corresponding density rises, exists not only in urban areas but also starts to take place on motorways or freeways more and more often, in particular behind merge or diverge points. Since the density in a hypercongested area is higher than the corresponding critical density, traffic has to slow down, which makes the flow rate lower than the capacity (McDonald et al. 1999; Verhoef, 2001; Small and Chu, 2003). This lengthens travelers' journey times, deteriorates traffic pollution, as well as makes the road resource under-utilized. As an undesired traffic phenomenon, traffic hypercongestion has become a problem we have to face in the developing countries as well as in the developed countries. Therefore, solving the problem has become a global challenge. This paper is concerned with it but mainly focuses on traffic hypercongestion behind freeway merges. The method used for this research is not empirical analysis based on field observations or data but involved in modeling freeway traffic flow, including merging and diverging.

Laval and Leclercq (2010) offered a model to approximate the vehicular traffic flow congestion dynamics on freeway corridors, which includes a conservation equation with a spatially continuous source term that represents net inflow from ramps. Clearly, the term is a function of time $t$ and location $x$. This model mathematically allows traffic to enter or leave a freeway simultaneously at the same location $x$ at time $t$. The core concern of this reference is the treatment of boundary conditions at merges on freeways. Different from the assumption that entry flows from on-ramps have full priority (used in e.g. Bayen et al. 2004; Coclite et al. 2005; Bastin et al. 2007), Laval and Leclercq (2010) followed their previous work in Laval and Leclercq (2008) and assumed that the actual inflow to a freeway from an on-ramp is a function of both the demand from the on-ramp and the flow on the freeway. The feasibility of this assumption was rooted in the finding from the observations of driver turn-taking behavior in congested freeway merges (Cassidy and Ahn 2005) that the percentage of the available freeway capacity allocated to competing flows from on-ramps is around a merge ratio. Laval and Leclercq (2010) proposed a set of boundary conditions for the treatment of flows at merges but it is found out that the merging scheme given in this reference can not always ensure that the sum of the on-ramp flow entering actually the freeway plus the freeway flow going actually downstream through a merge not greater than the freeway capacity at the merge.

On the basis of a thorough investigation of existing distribution schemes of determination of flows going through freeway merges in a supply-demand framework, Jin and Zhang (2003) proposed a simple distribution scheme satisfying the "fairness" condition, which requires that the flow that is eventually able to go through a merge be proportional to its corresponding demand upstream. This is consistent with the finding in Cassidy and Ahn (2005) and the assumptions in Laval and Leclercq (2008, 2010). Jin and Zhang (2003) has made a detailed comparison between this scheme and the merging models in Daganzo (1995) and Lebacuqe (1996). They took a further step to extend this scheme for merges with more than two feeding links. A key property of this distribution scheme is that the sum of the actual feeding flows that go actually through a merge can be guaranteed not to be greater than the available capacity at the merge. Therefore, in order to investigate traffic hypercongestion on freeways, this paper is to embed the distribution scheme for merging flows into the model proposed in Laval and Leclercq (2010). This is one of the intellectual merits of this paper.

Laval and Lecqlercq (2010) models traffic flow on "a long $n$-lane freeway corridor of length $L$ with entrances and exits evenly spaced $\delta$ distance units aparts." For this investigation, we chose a homogeneous freeway segment which contains an on-ramp and an off-ramp and the off-ramp lies upstream, which is a special case of the one investigated in Laval and Leclercq (2010), in which all on/off-ramps but the two have zero inflow/outflow rates. It is assumed that neither traffic incidents took place nor traffic control facilities existed on this segment of the freeway or on these ramps. The capacity of each facility is also assumed to remain the same over the time horizon under investigation. This scenario may correspond to

\footnotetext{
${ }^{2}$ If we treat the flow-density function as a triangular fundamental diagram, hypercongestion can be considered to be the same as congestion and both correspond to those states on the right branch of the diagram. If the left branch of the flow-density curve is nonlinear, a point on the left branch also corresponds to a congested but light congested state, where the flow rate increases as the speed falls. In this situation, a state on the right branch is often considered to be hypercongested (see Figure 3.1 on Page 71 in Small and Verhoef, 2007).
} 
a typical part of a real-life transportation system where a freeway passes by a city, with an off-ramp lying upstream towards the city and an on-ramp situated in the downstream from the city. The off-ramp and the on-ramp both serve the city and traffic hypercongestion may often happen on the freeway between the two ramps; sometimes the hypercongestion may even affect the flow leaving the freeway via the off-ramp when it grows upwards beyond the diverge or approach of the off-ramp. We can imagine that, if the city is a place with an abundant supply of employment positions, the morning (evening) demand flow coming from the freeway off-ramp into the city will be highly likely to be higher (lower) than the morning (evening) traffic flow departing the city via the freeway on-ramp. On the contrary, if the city is mainly a residential place, the morning (evening) demand flow coming from the freeway off-ramp will be highly likely to be lower (higher) than the morning (evening) traffic flow departing the city via the freeway on-ramp. In this paper, we will build an abstract linkage of the on-ramp and off-ramp flow profiles to the generated and attracted demand of the city the two ramps serve for, so that we may analyze how the generated and attracted demand of the city may affect traffic hypercongestion on the chosen freeway segment. This is a second intellectual merit of this paper. The other contribution of this paper includes those new findings obtained from a series of numerical experiments.

The rest of this paper is organized as follows. Next section presents the methodology to be used in this investigation, which is based on the freeway congestion dynamics in Laval and Leclercq (2010) but with a modified distribution scheme of freeway merging flows that has certain desirable properties. Section 3 analyzes traffic hypercongestion on the chosen freeway segment using this methodology while the entry flow from the upstream approach of the freeway was assumed to be constant within the time horizon and both deterministic and random on-ramp inflow rates were used. It consists of two sets of numerical experiments. The first set was carried out under the assumption that the upstream off-ramp was closed or traffic leaving the freeway via the off-ramp was too little to be worth counting. The first purpose of presenting this set is to show that those traffic phenomena identified in Laval and Leclercq (2010) can be reproduced using the modified method given in this paper. The other purpose is to show that a hypercongested area will start to formulate once the sum of the rate of flow from the on-ramp plus that from the upstream freeway is greater than the available capacity at the freeway merge and that it will start to shrink and eventually to disappear as the freeway flow or on-ramp flow falls. This shows that this modified method can be used to investigate freeway traffic hypercongestion. The second set of numerical experiments is to show the impacts on traffic hypercongestion of the different profiles of flows, both on the on-ramp and on the off-ramp. Our attention was mainly paid to two things: One is that the tail of the hypercongested area may go beyond the diverge so it affects the flow leaving the freeway and the other is the effects on the hypercongested area of the (im)balance of on- and off-ramp flow profiles, which is linked to the generated and attracted demand of the city the two ramps serve for. Section 4 closes the paper with some concluding remarks.

\section{Methodology}

\subsection{Model}

Let us choose a special case of the scenario prescribed in Laval and Leclercq (2010) - a homogeneous $n$-lane freeway segment of length $L$ that includes an $m$-lane on-ramp and an off-ramp and the on-ramp lies upstream; and both ramps serve the same city by which the chosen freeway segment passes. The length of the on-ramp is set to $d$. To facilitate the analysis in this paper, it is assumed that no congestion takes place on the off-ramp within the time horizon of interest, which implies that the traffic on the freeway can leave via the off-ramp without any delay. Therefore, there is no point in considering the traffic on the off-ramp and no need for the information on the off-ramp length and the number of lanes on the off-ramp. It is noteworthy that, unless stated otherwise, those notations for the on-ramp (off-ramp) will have the superscript ${ }^{\text {on }}$ ( ${ }^{\text {fff }}$ ) or subscript on (off). It is also assumed that neither traffic incidents took place nor traffic control facilities occurred within the time horizon of interest along the chosen part of the freeway. Another assumption is that no random events took place on the chosen part of the freeway within the chosen time horizon (i.e. supply is deterministic). 
A homogeneous equilibrium relationship exists between flow $(q)$ and density $(k)$ along the freeway segment and is also known as the homogeneous fundamental diagram (FD). It is written as follows:

$$
q(x, t)=q(k(x, t))
$$

which also applies to traffic flow on the on-ramp. According to the continuum traffic flow theory, the traffic conservation law along the freeway and on the on-ramp can be expressed as:

$$
\frac{\partial k}{\partial t}+\frac{\partial q}{\partial x}=g(t, x)
$$

where $g(t, x)$ denotes the net inflow rate. Generally, $g(t, x)=0$ unless $x=x_{\text {on }}$ and $x=x_{\text {off }}$ where $x_{\text {on }}$ and $x_{\text {off }}$ are respectively the positions where the on-ramp joins and the off-ramp leaves the freeway. Since here $x$ is a continuous location variable, $x_{\text {on }}\left(x_{\text {off }}\right)$ actually falls in an interval of locations on the freeway which corresponds to the width of the physical exit (approach) of an on-ramp (off-ramp), in particular when more than one lane exists on the on-ramp (off-ramp).

To determine $g(t, x)$ at the merge or diverge, we introduce two additional physical quantities that respectively represent the rate of inflow from an on-ramp and the rate of outflow from an off-ramp and denoted as $\phi^{+}(t, x)$ and $\phi^{-}(t, x)$ (in vehicles per unit time per unit distance). So $g(t, x)$ can be expressed as:

$$
g(t, x)=\phi^{+}(t, x)-\phi^{-}(t, x)
$$

Clearly, in real-life freeway traffic, $\phi^{+}(t, x)=0$ for all $x \neq x_{\text {on }}$ and $\phi^{-}(t, x)=0$ for all $x \neq x_{\text {off }}$. From now onwards, if all notation in the same equation have the same set of time and location, i.e. $(t, x)$, we will omit the time and location dimensions unless this might cause confusion.

Figure 1 illustrates the topological structure of the merge considered in the paper, where $\mu$ represents

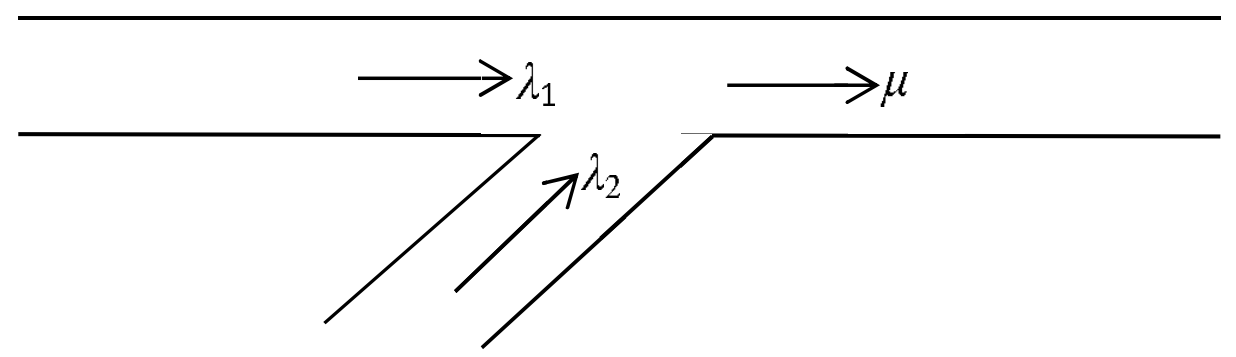

Figure 1: An abstract topological structure of a freeway segment with an on-ramp

the available capacity exactly at the merge and $\lambda_{1}$ and $\lambda_{2}$ denote the rates of the two flow streams towards the merge, respectively from the immediate upstream of the merge and from the on-ramp. In the demand-supply framework, as discussed in Jin and Zhang (2003), $\mu$ represents the supply (receiving capacity) while $\lambda_{1}$ and $\lambda_{2}$ are the demand (sending flow). Besides, let $q_{1}$ and $q_{2}$ denote the outflows coming from the two streams behind the merge point and actually flowing through it, and $q$ the total flow that actually passes through the merge. Then, we have $q=q_{1}+q_{2}$. Clearly, $q=\min \left\{\lambda_{1}+\lambda_{2}, \mu\right\}$. Then, Eq. (4) in Laval and Leclercq (2010) can be rewritten as:

$$
\begin{gathered}
q_{2}=\min \left\{1, \frac{\mu}{\lambda_{1}}\right\} \lambda_{2}, \quad \text { and } \\
q_{1}=q-q_{2} .
\end{gathered}
$$

Now let us look at two propositions on this scheme.

Proposition 1. When the demand from the upstream of a freeway merge is not greater than the available capacity at the merge, the merging scheme in Eq. (4) gives the full priority to the demand from the on-ramp.

Proof. When the condition in the above property is true, i.e. $\lambda_{1} \leq \mu$, then Eq. (4a) gives $q_{2}=\lambda_{2}$, which implies that all demand from the on-ramp will be allowed to enter the freeway. In other words, given this condition, the full priority is given to the flow from the on-ramp. 
Remark 1. We have three cases to look at. First, $\lambda_{1}+\lambda_{2} \leq \mu$, which gives $q_{1}=\lambda_{1}$ and $q_{2}=\lambda_{2}$. The above distribution scheme (4) is not a problem. Second, $\lambda_{1}+\lambda_{2}>\mu$ with $\lambda_{1}>\mu$, which yields $q_{2}=\frac{\lambda_{2}}{\lambda_{1}} \mu$ and $q_{1}=\left(1-\frac{\lambda_{2}}{\lambda_{1}}\right) \mu$. Clearly, this solution is infeasible when $\lambda_{2}>\lambda_{1}$ is true; but the thing is that we can not exclude the occurrence of $\lambda_{2}>\lambda_{1}$. Third, $\lambda_{1}+\lambda_{2}>\mu$ with $\lambda_{1} \leq \mu$, which gives $q_{2}=\lambda_{2}$ and $q_{1}=\mu-\lambda_{2}$. Again, this will be an infeasible solution when $\lambda_{2}>\mu$ turns to be true. The other problem with the third case is that the merging scheme in Eq. (4) gives the full priority to the on-ramp flow. Therefore, the property of the scheme in Eq. (4) given in Proposition 1 is undesirable.

Proposition 2. Based on the scheme in Eq. (4), the resulting flow actually entering from an on-ramp may be greater than the downstream available capacity.

Proof. Look at two situations. First, when $\mu \geq \lambda_{1}$ is true, Eq. (4a) gives $q_{2}=\lambda_{2}$. If $\lambda_{2}>\mu$ then $q_{2}>\mu$. This means that this property is true.

Second, $\mu<\lambda_{1}$ plus Eq. (4a) results in $q_{2}=\frac{\lambda_{2}}{\lambda_{1}} \mu$. If $\lambda_{2}>\lambda_{1}$ then $q_{2}>\mu$, which also means that the resulting flow actually entering from an on-ramp may be greater than the available capacity at the merge.

Remark 2. All conditions mentioned in the two propositions or their proofs can occur in real-life traffic. The condition in Property 1 will be true when the upstream density is not greater than that at the merge, which often happens due to the entry of on-ramp traffic to the freeway. Second, the conditions in the first part of the proof of Proposition 2, i.e. $\mu \geq \lambda_{1}$ and $\lambda_{2}>\mu$, say that the available capacity at the merge is not less than the demand from the upstream but less than the demand from the on-ramp. This can happen although it may be that often. Third, the conditions in the second part of the proof of Proposition 2, i.e. $\mu<\lambda_{1}$ and $\lambda_{2}>\lambda_{1}$, mean that the demand from the upstream of the merge is greater than the available capacity at the merge but less than the demand from the on-ramp. This can also take place on freeways.

Remark 3. Since all conditions mentioned in the two propositions or their proofs can occur in real-life traffic and the above two properties of the merging scheme in Eq. (4) given in the two propositions are undesirable, it is improper to use the scheme to model the merging operations.

To avoid the use of the scheme given in Eq. (4) due to its above two undesirable properties, we took a merging scheme proposed in Jin and Zhang (2003) that can avoid the full priority allocated to on-ramp flows and can ensure that the resulting total traffic flow from the upstream of the freeway or from the on-ramp does not exceed the capacity at the merge. In Jin and Zhang (2003), the rate of actual inflow from the on-ramp at $x_{\mathrm{on}}$ is expressed as:

$$
\phi^{+}\left(t, x_{\mathrm{on}}\right)=\frac{\lambda^{x_{\mathrm{on}}}\left(k^{\mathrm{on}}(t, d)\right)}{\lambda\left(k\left(t, x_{\mathrm{on}}^{-}\right)\right)+\lambda^{x_{\mathrm{on}}}\left(k^{\mathrm{on}}(t, d)\right)} \min \left\{\lambda\left(k\left(t, x_{\mathrm{on}}^{-}\right)\right)+\lambda^{x_{\mathrm{on}}}\left(k^{\mathrm{on}}(t, d)\right), \mu\left(k\left(t, x_{\mathrm{on}}^{+}\right)\right)\right\} / \delta
$$

where $x_{\mathrm{on}}^{+}$and $x_{\mathrm{on}}^{-}$respectively denote the points immediately after and before the on-ramp, $\mu$ and $\lambda$ are respectively the supply (receiving) and demand (sending) functions that are introduced in Lebacque (1996) for the kinematic wave theory of traffic flow, $\lambda^{x_{\mathrm{on}}}$ is the function of demand flow from the on-ramp and $\delta$ is the length of an insertion section where vehicles can carry out lane-changing from the on-ramp to the freeway. Jin and Zhang (2003) has shown that this scheme is equivalent to the merging models in Daganzo (1996) and Lebacque (1996).

Laval and Leclercq $(2010)$ assumed that $\phi^{-}\left(t, x_{\text {off }}\right)$ is a time-varying function of freeway flow arriving at the off-ramp. In the existing literature on modeling traffic flows on freeways, $\phi^{-}\left(t, x_{\mathrm{off}}\right)$ is assumed to be exogenous unless connected roads are simultaneously considered. We will make our further assumptions on the function later for our numerical analysis.

In summary, $g(t, x)$ is defined in $[0, L] \times[0,+\infty)$ and it is nonzero only when an on-ramp or off-ramp exists in the special case of the scenario prescribed in Laval and Leclercq (2010). More specifically, we have

$$
g(t, x)= \begin{cases}\phi^{+}\left(t, x_{\mathrm{on}}\right), & \text { if } x=x_{\mathrm{on}}, \\ -\phi^{-}\left(t, x_{\mathrm{off}}\right), & \text { if } x=x_{\mathrm{off}}, t \in[0,+\infty) \\ 0, & \text { otherwise }\end{cases}
$$


We assume, without loss of generality, that the freeway is empty at $t=0$ and that there is a flow rate equal to $\lambda_{0}(t)$ that arrives at the upstream end of the freeway segment. Thus, the initial and boundary conditions for the freeway can be given:

$$
\begin{array}{ll}
k(0, x)=0, & x \in[0, L] \\
\lambda(t, 0)=\lambda_{0}(t), & t \in[0,+\infty)
\end{array}
$$

The boundary condition on the demand at the upstream end of the freeway segment is given in the form of flow $\lambda_{0}(t)$ other than density $k(t, 0)$, which avoids creating an ill-posed scenario when a queue spills back beyond the end of the upstream end of the freeway segment under investigation. In the literature it is often to see the demand given in the form of flow rates, such as in Lebacque and Khoshyaran (2005), Ge and Zhou (2012) and Carey and Ge (2012). In addition, the Riemann boundary condition is imposed on the downstream boundary of the freeway segment, i.e. the spatial derivative of traffic density at the downstream boundary is assumed to be zero, which means that the vehicles arriving at the downstream end of the freeway segment can leave with no delay. This condition also applies to the approach to the off-ramp.

The following initial and boundary conditions are for the continuous-time and continuous-space flow on the on-ramp:

$$
\begin{array}{ll}
k^{\text {on }}(0, y)=\alpha\left(0, x_{\text {on }}\right) \delta / u_{\text {on }}, & \forall y \in[0, d] \\
\lambda^{\text {on }}(t, 0)=\lambda_{\text {on }}(t), & \forall t \in[0,+\infty)
\end{array}
$$

where $u_{\mathrm{on}}$ is the free flow speed on the on-ramp and $\alpha\left(t, x_{\mathrm{on}}\right)$ is the demand rate (in vehicles per unit time per unit distance) representing the flow waiting on the on-ramp to enter the freeway but not the resulting flow that actually enters the freeway.

To sum up, the only difference of the model we will use later from one in Laval and Leclercq (2010) lies in the treatment of flows at the merge, specifically, it no longer uses the merging scheme in Eq. (4) but one in Eq. (5). This modification makes the resulting entry flow from an on-ramp plus the flow from the freeway upstream that actually goes through the corresponding merge not greater than the available capacity at the merge and the on-ramp flow not receive the full priority when the available capacity at the merge can not meet the total demand from the upstream on the freeway and from the on-ramp.

\subsection{Solution method}

\subsubsection{The classical method for solving the LWR model}

The intrinsic connotation of the kinematic wave model of traffic flow is that it is a hyperbolic system of conservation law. A classical method for solving (2) uses the method of characteristics, which is the foundation of solving hyperbolic partial differential equations (HPDE). A shock arises in the hyperbolic system when its characteristics intersect, hence multiple-valued solutions occur at the same point $(t, x)$. A requirement for the existence of a unique single-valued solution is that the solution should satisfy the "entropy" condition (Rainer 1990); Newell (1993) independently gave another method to get the single-valued solution in the sense of engineering without proving. On the other hand, under the socalled "Rankine-Hugoniot" jump condition (Rankine, 1870; Hugoniot, 1887 1889), one can obtain the equation of shockwave interfaces. However, given a general set of initial and boundary conditions, it is very difficult, if not impossible, to express in analytical form the solution to a set of partial differential equations capturing traffic dynamics on the freeway and on the ramps. Therefore, it is not our intention to use the classical method to solve the model presented in the previous section. In fact, it is quite natural to use an approximate solution solution for them.

\subsubsection{A Godunov method}

This subsection is to give a discretization scheme for solving the set of partial differential equations (2) for traffic dynamics on the freeway and on the on-ramp. An efficient numerical method for solving this type of models is one proposed in Godunov (1959). In the Godunov method, a link is partitioned into small cells of length $\triangle x$ and the time horizon of interest is divided into homogeneous time intervals of 
duration $\triangle t$. This scheme requires that the space step size and the time step size satisfy the CourantFriedrichs-Lewy or CFL condition (Courant et al. 1928), which ensures that no vehicle can cross both the upstream and downstream boundaries of a cell within the same time interval; the CFL condition can be mathematically written as follows:

$$
\triangle x \geq v_{f} \triangle t
$$

where $v_{f}$ is the link's free flow speed.

\section{1) Discretizing freeway segment}

For a freeway segment, Eq. (2) can be approximated by the following finite difference equations

$$
\frac{k_{i}^{t+1}-k_{i}^{t}}{\triangle t}+\frac{q_{i}^{t}-q_{i-1}^{t}}{\triangle x}=g_{i}^{t}
$$

where $k_{i}^{t}$ denotes the density in cell $i$ at the beginning of time interval $t, q_{i}^{t}$ represents the average rate of flow leaving cell $i$ and entering cell $i+1$ during interval $t$ and $g_{i}^{t}$ denotes the average rate of net flow entering cell $i$ during interval $t$ and is given by

$$
g_{i}^{t}= \begin{cases}\phi_{i}^{+t}, & \text { if cell } i \text { is a merge cell } \\ -\phi_{i}^{-t}, & \text { if cell } i \text { is a diverge cell } \\ 0, & \text { if cell } i \text { is an ordinary cell }\end{cases}
$$

in which $\phi_{i}^{-t}$ and $\phi_{i}^{+t}$ denote respectively the average rate of flow leaving cell $i$ via the off-ramp and that entering cell $i$ from the on-ramp during time interval $t$.

In numerical computing, suppose that we have reached this iteration in which we have obtained the values of $k_{i}^{t}, q_{i-1}^{t}$ and $g_{i}^{t}$ (or $\phi_{i}^{-t}$ and $\phi_{i}^{+t}$ ) in Eq. (10). Then, once we have the value of $q_{i}^{t}$, it is a straightforward to use Eq. (10) to get the value of $k_{i}^{t+1}$. The following proposition gives how to obtain the value of $q_{i}^{t}$ :

Proposition 3. The average rate of flow leaving cell $i$ and entering cell $i+1$ during interval $t$ is given by

$$
q_{i}^{t}= \begin{cases}\min \left\{\lambda\left(k_{i}^{t}\right), \mu\left(k_{i+1}^{t}\right)-\phi_{i+1}^{+t} \Delta x\right\} & \text { if } i \text { and } i+1 \text { are respectively an ordinary and a merge cell } \\ \min \left\{\lambda\left(k_{i}^{t}\right)-\phi_{i}^{-t} \Delta x, \mu\left(k_{i+1}^{t}\right)\right\} & \text { if } i \text { and } i+1 \text { are respectively a diverge and an ordinary cell } \\ \lambda\left(k_{i}^{t}\right) & \text { if } i \text { is the last cell on the chosen freeway segment } \\ \min \left\{\lambda\left(k_{i}^{t}\right), \mu\left(k_{i+1}^{t}\right)\right\} & \text { otherwise }\end{cases}
$$

where $\phi_{i}^{-t}$ is given in advance and

$$
\phi_{i+1}^{+t}=\frac{\lambda^{x_{\mathrm{on}}}\left(k_{M}^{\mathrm{on} t}\right)}{\lambda\left(k_{i}^{t}\right)+\lambda^{x_{\mathrm{on}}}\left(k_{M}^{\mathrm{on} t}\right)} \min \left\{\lambda\left(k_{i}^{t}\right)+\lambda^{x_{\mathrm{on}}}\left(k_{M}^{\mathrm{on} t}\right), \mu\left(k_{i+1}^{t}\right)\right\} / \Delta x \quad(M \text { is the last cell of the on-ramp }) .
$$

Remark 4. This proposition is a summary of those results given in many relevant papers, hence we no longer give a proof of it here.

Remark 5. It should be noted that the above proposition does not cover the situation in which two successive cells correspond to two ramps. We did not intend to discuss the situation because it rarely occurs on real-life freeways.

Remark 6. For the convenience of exposition, it is implicitly assumed in Eqs (12)-(13) that the exit of the on-ramp exactly corresponds to one cell only. Under this assumption, $\Delta x=\delta$ is true.

2) Discretizing on-ramp

For the on-ramp part, Eq. (2) can be approximated with a finite difference equation below:

$$
\frac{k_{j}^{\text {ont } t+1}-k_{j}^{\text {on } t}}{\Delta t}+\frac{q_{j}^{\text {ont }}-q_{j-1}^{\text {ont }}}{\Delta y}=0, \quad \forall \text { cell } j
$$


where $q_{j}^{\text {ont }}$ and $q_{j-1}^{\text {ont }}$ have the same meanings as $q_{i}^{t}$ and $q_{i-1}^{t}$. Since Eq. (14) has no source term, it is straightforward to give an equation to determine $q_{j}^{\text {ont }}$, i.e.

$$
q_{j}^{\mathrm{on} t}=\min \left\{\lambda^{x_{\mathrm{on}}}\left(k_{j}^{\mathrm{on} t}\right), \mu^{x_{\mathrm{on}}}\left(k_{j+1}^{\mathrm{ont}}\right)\right\}
$$

A special case is that cell $j$ is the last cell of the on-ramp, i.e. $j=M$. If the number of the corresponding merge cell on the freeway is $i+1$ then $q_{j}^{\text {ont }}=\phi_{i+1}^{+t}$ where $\phi_{i+1}^{+t}$ is determined by Eq. (13).

\section{Applications}

The methodology discussed in the previous section is for a general FD. Here, in order to compare our results to those in Laval and Leclercq (2010), we also consider triangular fundamental diagrams (FDs) whereas the FDs for flows on the freeway and on the on-ramp have different parameter values. For the freeway, the free flow speed is $u_{e}=100 \mathrm{~km} /$ hour, wave speed $w_{e}=25 \mathrm{~km} /$ hour, and jam density $\kappa_{e}=n \kappa$, where $\kappa=180 \mathrm{veh} / \mathrm{km}$ is the jam density of a single lane that is the same for the freeway and for the on-ramp. Following Daganzo (1994), the receiving and sending functions for the flow on the freeway are given below

$$
\begin{aligned}
\mu(k) & =\min \left\{w_{e}\left(\kappa_{e}-k\right), Q_{e}\right\} \\
\lambda(k) & =\min \left\{u_{e} k, Q_{e}\right\}
\end{aligned}
$$

where $Q_{e}=u_{e} w_{e} \kappa_{e} /\left(w_{e}+u_{e}\right)$ is the capacity of the freeway under investigation.

For the on-ramp, the free flow speed is $u_{\mathrm{on}}=84 \mathrm{~km} /$ hour, the wave speed $w_{\text {on }}=21 \mathrm{~km} /$ hour, and the jam density $\kappa_{\mathrm{on}}=m \kappa$. Following Daganzo (1994), the receiving and sending functions for the flow on the on-ramp can be written in the same form as Eq. (16), i.e.

$$
\begin{aligned}
& \mu^{x_{\text {on }}}\left(k^{\text {on }}\right)=\min \left\{w_{\text {on }}\left(\kappa_{\text {on }}-k^{\text {on }}\right), Q_{\text {on }}\right\} \\
& \lambda^{x_{\text {on }}}\left(k^{\text {on }}\right)=\min \left\{u_{\text {on }} k^{\text {on }}, Q_{\text {on }}\right\}
\end{aligned}
$$

where $Q_{\mathrm{on}}=u_{\mathrm{on}} w_{\mathrm{on}} \kappa_{\mathrm{on}} /\left(w_{\mathrm{on}}+u_{\mathrm{on}}\right)$ is the capacity of the on-ramp.

It can be readily derived that the critical density on the freeway is $\kappa_{e c}=0.2 \kappa_{e}$ and that on the on-ramp $\kappa_{\text {onc }}=0.2 \kappa_{\text {on }}$. It is also assumed that there are four lanes on the freeway while two lanes on all ramps, i.e. $n=4$ and $m=2$; hence, $\kappa_{e}=n \kappa=720 \mathrm{veh} / \mathrm{km}$ and that on the on-ramp $\kappa_{o n}=m \kappa=360 \mathrm{veh} / \mathrm{km}$. Figure 2 illustrates the two FDs used for our numerical experiments in this section.

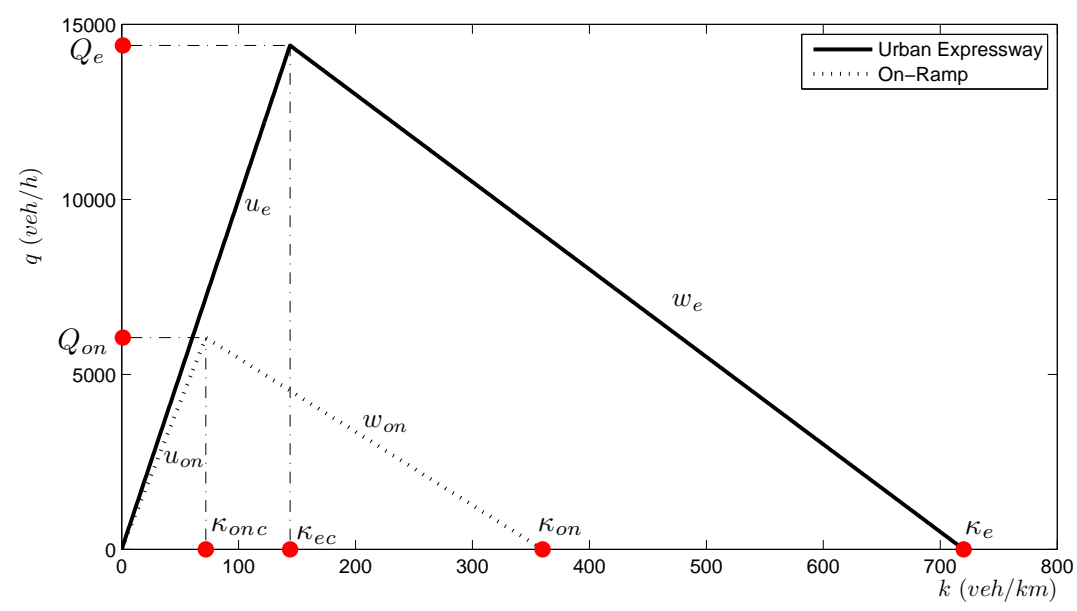

Figure 2: The triangular fundamental diagram FDs) for the chosen freeway and on-ramp

The time horizon used for this investigation is $[0, T]$ where $T=60 \mathrm{~min}$ and the time step size used is $\triangle t=3.6 \mathrm{sec}$. Given the CFL condition, to simplify our treatment at the exit or entry of a ramp, we 
set the length of a cell to the length of a merging section, i.e. $\triangle x=\Delta y=\delta=100 \mathrm{~m}$, respectively for the cells on the freeway and on the on-ramp. Here are the other parameter values: $L=20 \mathrm{~km}, d=2 \mathrm{~km}$, $\gamma=0.18 \kappa_{e}=0.9 \kappa_{e c}$. In addition, $x_{\mathrm{off}}=4 \mathrm{~km}$ and $x_{\mathrm{on}}=5 \mathrm{~km}$, which implies that the on-ramp position is $5 \mathrm{~km}$ away from the upstream end of the freeway segment while the off-ramp is $1 \mathrm{~km}$ upstream from the on-ramp.

As mentioned previously, $\phi^{-}\left(t, x_{\text {off }}\right)$ is usually an exogenous variable. In this research, it is assumed that, if cell $i$ is a diverge cell then $\phi_{i}^{-t}$ is determined in the following manner

$$
\phi_{i}^{-t}= \begin{cases}0, & \text { if } \lambda\left(k_{i}^{t}\right)<\alpha(t) \delta \\ \theta \alpha(t) \delta, & \text { otherwise }\end{cases}
$$

where $\alpha(t) \delta$ represents the average flow entering the on-ramp during the interval $t$. This relationship may not be true in reality but it is not the purpose of this paper to investigate an exact temporal relationship between the profiles of flow entering from an on-ramp and flow leaving the freeway via the closest upstream off-ramp if it does exist. We only used the assumed relationship in Eq. (18) to illustrate how the balance or imbalance of on-ramp entry flow and off-ramp exit flow can affect traffic hypercongestion on the chosen freeway segment, where their balance is represented by the parameter $\theta$.

As mentioned previously, the primary purpose of this paper is to investigate traffic hypercongestion on freeways between an on-ramp and its immediate upstream off-ramp and the method used for this investigated is based on one in Laval and Leclercq (2010) but its merging scheme was replaced with one proposed in Jin and Zhang (2003) to remove its identified shortcomings. It is assumed in this reference that traffic can enter or leave the freeway anywhere anytime whereas we assumed that traffic can only enter from the upstream end of the freeway segment and the on-ramp and exit from the off-ramp and the downstream end of the freeway segment. It is interesting to see that, given our settings, those phenomena presented in Laval and Leclercq (2010) can also be observed here. We first experimented with $\theta=0$, which means that the off-ramp of the chosen freeway segment has been closed or may correspond to a situation where traffic leaving the freeway via the off-ramp was too little to be worth counting. Then we moved on to investigate traffic hypercongestion on the chosen freeway segment as $\theta$ varies from 0 to 0.35 , then to 0.65 and finally to 1.

In the later analysis, we also aimed to build an abstract linkage between the on-ramp and off-ramp flow profiles and the generated and attracted demand of the city the two ramps served for. This linkage enabled us to analyze how the balance between the generated and attracted demand of the city may affect traffic hypercongestion on the chosen freeway segment.

\subsection{Off-ramp was closed while on-ramp was open}

This may correspond to a very special case in which the attracted demand of the city and its adjacent area the off-ramp served was zero or too little to be worth counting. Another relevant scenario is that a city or town is served only by an on-ramp and that no any other ramps exist in a few miles upstream and downstream from the on-ramp. We can consider such a city and its adjacent area is purely a residential place. Some satellite cities of a metropolitan city can be like this; a place like this may well be an area composed of a number of villages close to each other. In the morning rush hour, many people from a satellite city and its adjacent area travel to the metropolitan city to work whereas only an extremely low demand travels to the satellite city and its adjacent area.

This subsection first investigates a case whose profile of inflow from the on-ramp was constant. Subsequently, we compare the results from 3 different on-ramp inflow profiles: 1) constant flow rates (i.e. the first case), 2) flow rates that increase first, remain at a peak level for a while and then decrease (an arch-shaped profile), 3) flow rates that decrease first, stay flat for a while and then increase (a valleyshaped profile). Last, we look at a case in which the flow rates fluctuate around a constant inflow profile in a uniformly-distributed manner.

\subsubsection{Constant on-ramp inflow rates}

Numerical analysis 
This case means that

$$
\alpha\left(t, x_{\mathrm{on}}\right)=a \quad \forall t \in[0, T] .
$$

Unless stated otherwise, we assume $a=50,000 \mathrm{veh} / \mathrm{hr} / \mathrm{km}$. Figure 3(a) shows the density contour map corresponding to this case, in which 6 regions can be clearly identified and they are separated by the characteristic lines or shock waves. As shown in Figures 3(b)-(c), the density in each of these regions was identical. Clearly, the density in regions $A_{1}$ and $A_{2}$ was zero. Except that the traffic in region $\mathbf{D}$ suffered from traffic hypercongestion, the traffic in all other regions traveled at the free-flow speed; in other words, among the 6 regions, region $\mathbf{D}$ was the only one whose density was above the critical density. The traffic in regions $\mathbf{B}_{1}$ and $\mathbf{B}_{2}$ all came from the on-ramp. Point "1" refers to the location where the first vehicle arrived at the merge and it came from the on-ramp.

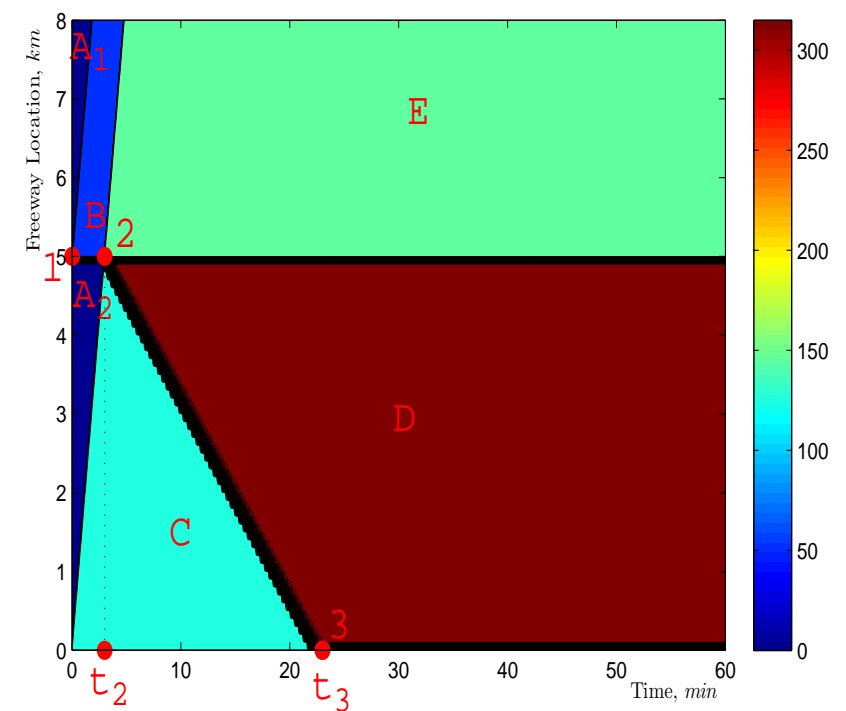

(a)

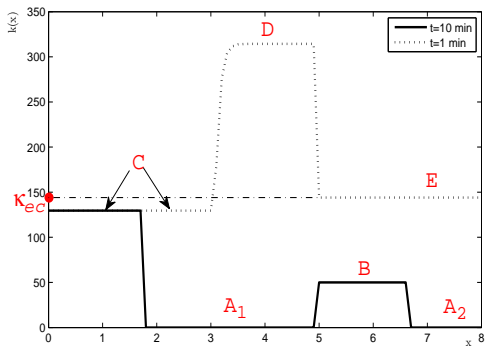

(b)

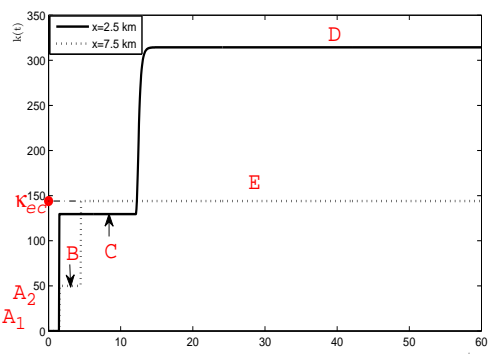

(c)

Figure 3: Numerical Solution for constant on-ramp inflow profile [(a) Density contour map for the freeway segment, (b) Density profiles respectively at $t=1 \mathrm{~min}$ and $10 \mathrm{~min}$ on the freeway, and (c) Density profiles respectively at $x=2.5 \mathrm{~km}$ and $7.5 \mathrm{~km}$ on the freeway.]

Point "2" marks the start of traffic hypercongestion, where, as can be seen on the density contour map, four characteristic curves met. The point is exactly the merge point of the freeway and the on-ramp. The hypercongestion occurred because the demand for flowing through the merge point was greater than its available capacity. In fact, at this point, the flow from the upstream and from the on-ramp were respectively $u_{e} \gamma=100 \times 0.18 \times 4 \times 180=12,960 \mathrm{veh} / \mathrm{hr}$ and $\alpha \delta=5,000 \mathrm{veh} / \mathrm{hr}$, which yields a sum equal to $17,960 \mathrm{veh} / \mathrm{hr}$ higher than the capacity of $u_{e} \kappa_{e c}=14,400 \mathrm{veh} / \mathrm{hr}$. Since the total demand from the upstream and from the on-ramp was unable to flow freely through the merge into the downstream, a traffic hypercongestion region started to form immediately behind the merge, i.e. region $\mathbf{D}$ in the density contour map in Fig. 3(a). The region grew longer and went beyond the upstream end of the freeway segment as time went by. It is interesting to see that the density in the hypercongested region $\mathbf{D}$ never reached the jam density and stayed at $k=314 \mathrm{veh} / \mathrm{km}$, which was above the critical density by $118.75 \%$ but lower than the jam density by $56.25 \%$. In reality, this may be a stop-and-go queue or a slow-moving traffic platoon whose head position remains unchanged and whose tail position depends on the arrival patterns of freeway upstream flow and on-ramp inflow.

Point "3" marks that the hypercongestion has been propagated backward and went beyond the upstream end of the freeway segment, which indicates that a local congestion at the merge point has led to the hypercongestion growing upstream continuously. This is an undesired traffic phenomenon. The 
shockwave or characteristic line $\mathbf{2}-\mathbf{3}$ captures the tail of the hypercongestion region. On one side, on the two sides of the merge, region $\mathbf{E}$ was undercongested whereas region $\mathbf{D}$ was hypercongested. On another hand, the traffic in both regions were in equilibrium.

In this case, the freeway flow rates was up to $90 \%$ of the freeway capacity. Although the on-ramp flow was only $34.72 \%$ of the freeway capacity, traffic hypercongestion took place behind the merge and grew upstream continuously. This is due to the fact that the sum of the two percentages was $124.72 \%$, which means that the demand was above the capacity at the merge by $24.72 \%$, hence the hypercongestion was inevitable. To resolve this problem, we may either reduce the inflow from the on-ramp or divert the freeway flow via an upstream off-ramp, which will be discussed in Subsection 3.2.

\section{Analytical Solution}

Since a triangular fundamental diagram is used, a state in each region in Figure 3 is either hypercongested or in a free-flow state and the speeds of characteristics associated with the two types of states are respectively $-w_{e}$ or $u_{e}$. Therefore, we can get an analytical solution for each region independently, which only depends on boundary conditions. In this case, $\phi^{+}\left(t, x_{o n}\right)$ takes only two possible values depending on the traffic state at the exit of the on-ramp, i.e.

$$
\phi^{+}\left(t, x_{o n}\right)= \begin{cases}a, & \text { (free-flow) }, \\ w_{e}\left(\kappa_{e}-k\left(t, x_{o n}^{+}\right)\right) Q_{o n} /\left(\left(Q_{e}+Q_{o n}\right) \delta\right), & \text { (hypercongested) } .\end{cases}
$$

Now look at the following simple hyperbolic equation:

$$
\frac{\partial k}{\partial t}+w \frac{\partial k}{\partial x}=0
$$

where $w=\mathrm{d} q / \mathrm{d} k$ is constant, and can be a positive or negative number. The general solution of Eq. (21) can be determined by:

$$
k(t, x)=f(x-w t)
$$

where $f$ is an arbitrary function. Additionally, if the boundary condition is known, i.e. $k(t, 0)=h(t)$, where $h$ is a given function then we have $f(-w t)=h(t)$, i.e. $f(t)=h(-t / w)$, which gives the analytical solution of Eq. (21) with the boundary condition $k(t, 0)=h(t)$ is $k(t, x)=h(t-x / w)$. A very special case is that $h(t)$ is constant, then $k(t, x)$ is also must be constant.

Table 1 lists the information required for solving the kinematic wave model inside each region in Figure 3 and the corresponding analytical expressions.

Table 1: The Information and the Analytical Expressions

\begin{tabular}{ccccc}
\hline Region & Freeway & $\mathrm{d} q / \mathrm{d} k$ & Boundary Condition & Analytical Expression \\
\hline $\mathrm{A}_{1}$ & No Vehicles & $\backslash$ & $\backslash$ & 0 \\
$\mathrm{~A}_{2}$ & No Vehicles & $\backslash$ & $\backslash$ & 0 \\
$\mathrm{~B}$ & Free- -flow & $u_{e}$ & $(w \delta) / u_{e}$ & $(w \delta) / u_{e}$ \\
$\mathrm{C}$ & Free-flow & $u_{e}$ & $\gamma$ & $\gamma$ \\
$\mathrm{D}$ & Hypercongestion & $-w_{e}$ & $\kappa_{e}-Q_{e}^{2} /\left(\left(Q_{e}+Q_{o n}\right) w_{e}\right)$ & $\kappa_{e}-Q_{e}^{2} /\left(\left(Q_{e}+Q_{o n}\right) w_{e}\right)$ \\
$\mathrm{E}$ & Critical Density & $u_{e}$ & $\kappa_{c}$ & $\kappa_{c}$ \\
\hline
\end{tabular}

In addition, $t_{2}$ and $t_{3}$ in Figure 3 can also be obtained analytically, i.e. $t_{2}=L_{1} / u_{e}$ and $t_{3}=t_{2}+L_{1} / w_{e}$, where $L_{1}$ is the distance between the upstream end of the freeway and the merge point.

\subsubsection{Traffic hypercongestion under three different on-ramp inflow profiles}

Figure 4 shows three profiles of inflow from the on-ramp, whose corresponding density contour maps are given in Figure 5. Here are the mathematical forms of these on-ramp inflow profiles: 


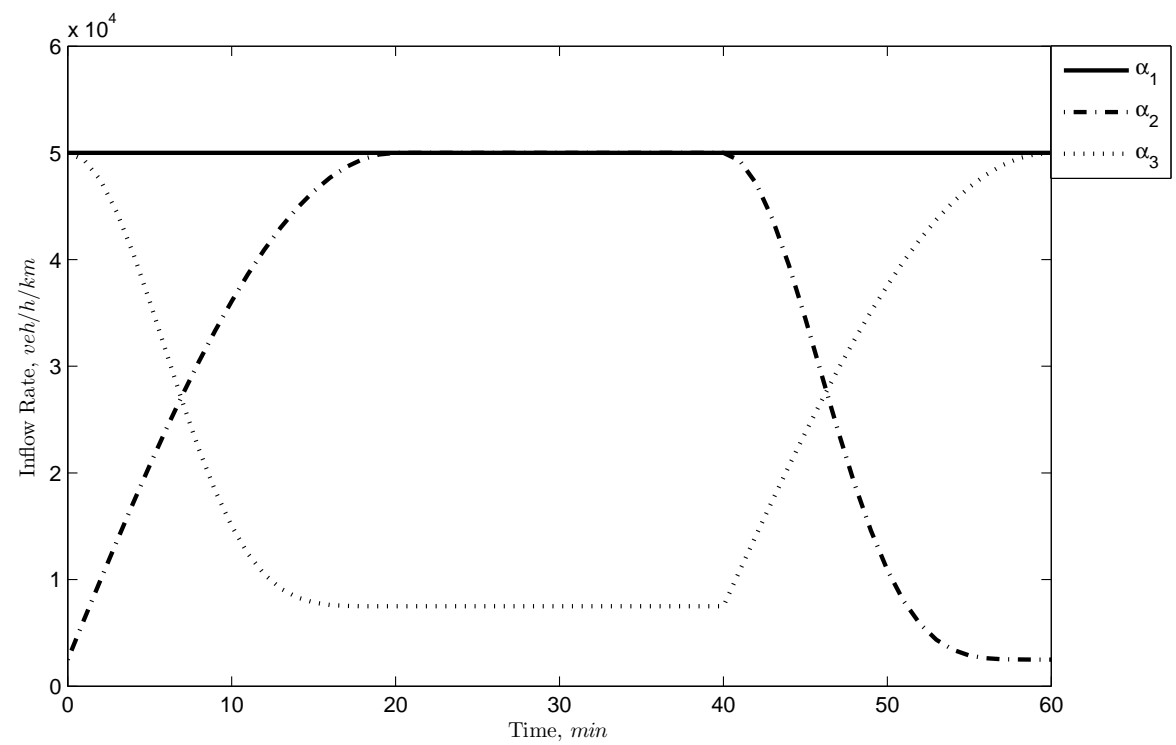

Figure 4: On-ramp inflow profiles

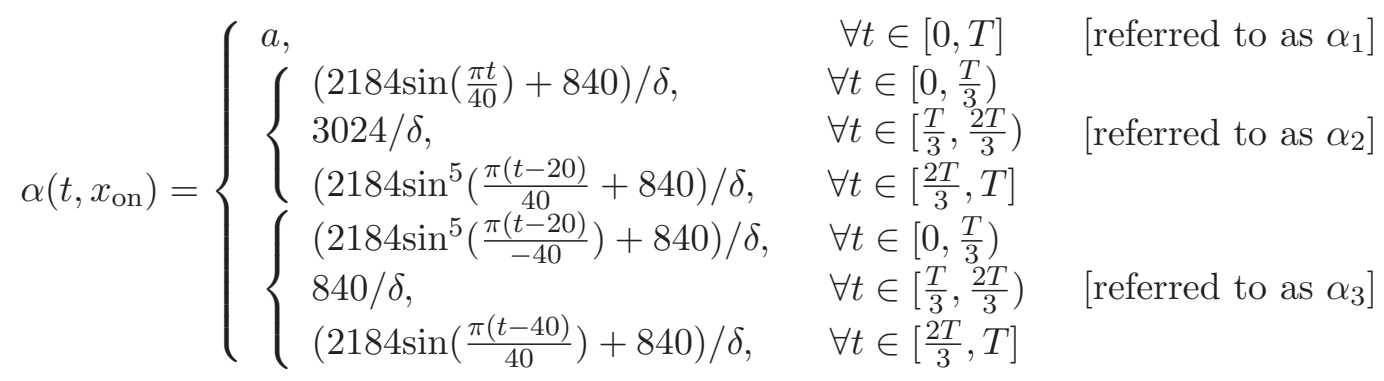

Profile $\alpha_{1}$ and its corresponding density contour map have been analyzed in the previous subsection. This flat on-ramp inflow profile resulted in a hypercongested area that kept growing upstream uniformly. The arch-shaped profile $\alpha_{2}$ has been used in Carey and Ge (2001) and their other work, which tends to represent a demand profile over a rush hour, rising first up to a flat level and then falling down. In addition, it can avoid sharp changes in flow rates. The valley-shaped profile $\alpha_{3}$ may correspond to a transitional period from one peak time to another.

It is easy to understand, can also be seen in Figure 5, that the first vehicle from the on-ramp in all three cases shared the same trajectory due to no effects of any downstream traffic on it. So did the first vehicle from the freeway upstream, which was attributed to two aspects. First, since the traffic from the on-ramp was very light so no hypercongestion occurred yet by the time when the first vehicle from the freeway upstream arrived at the merge point. Second, because of the use of a triangular FD, even though the rates of on-ramp inflow in the three cases were different, the traffic from the merge downwards was traveling at the same speed and equal to the free-flow speed.

In Figure 5(b), as the inflow from the on-ramp continued to increase, the hypercongestion started to form at $t=t_{2}$ (corresponding to point 2). From this time instant onwards, a hypercongestion region on the freeway started to grow upstream. The characteristic curve $\mathbf{2}-\mathbf{3}$ represents the tail of the hypercongested region and point " 3 " marks a moment that the hypercongestion has gone beyond the upstream end of the chosen freeway segment. The far-right side of region $\mathbf{D}$ in Figure 5(b) shows the dissipation of the hypercongestion while the far-left side of this region captures the formulation of the hypercongestion. From point 2 rightwards until the traffic was settled in equilibrium, the hypercongestion became gradually worse and worse because of the ever-increasing inflow rate from the on-ramp, as evidenced by the everincreasing density from the left to the right in this region (more specifically in region $\mathrm{D}_{1}$ ). Then, as the stable state was violated because of the fall of the on-ramp inflow rates, the traffic in the hypercongested region started to move faster, as shown by the shockwaves in region $\mathrm{D}_{3}$ of the density contour map and 


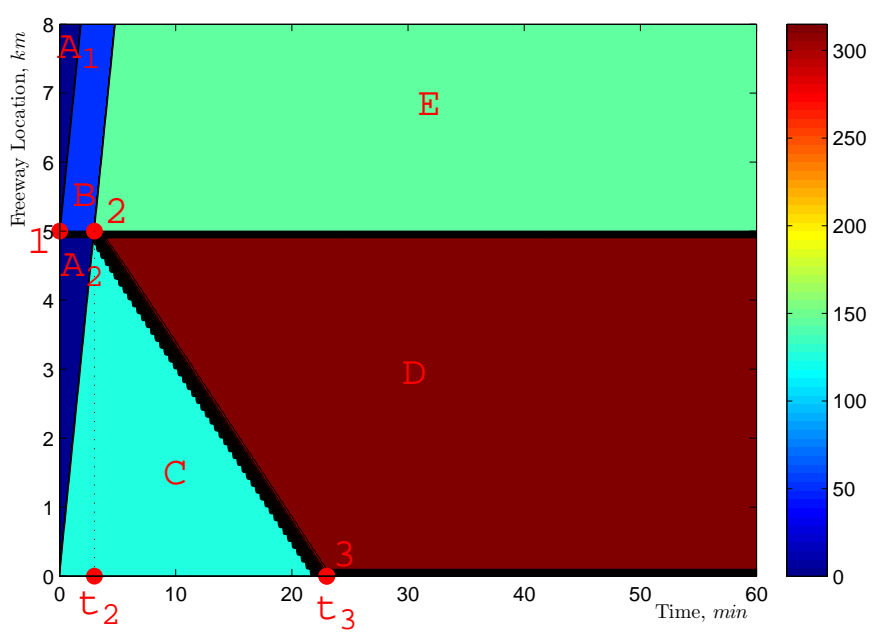

(a) Profile $\alpha_{1}$

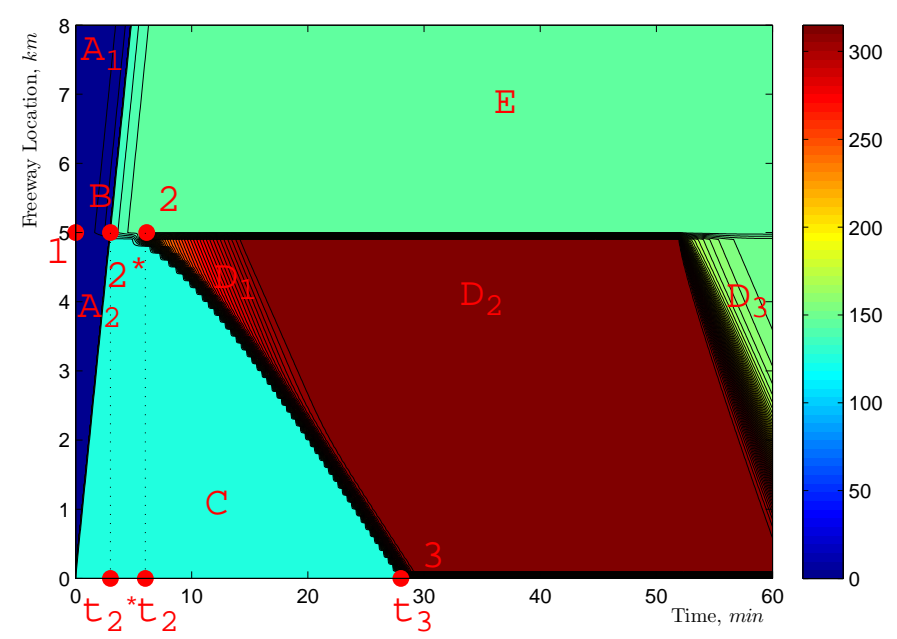

(b) Profile $\alpha_{2}$

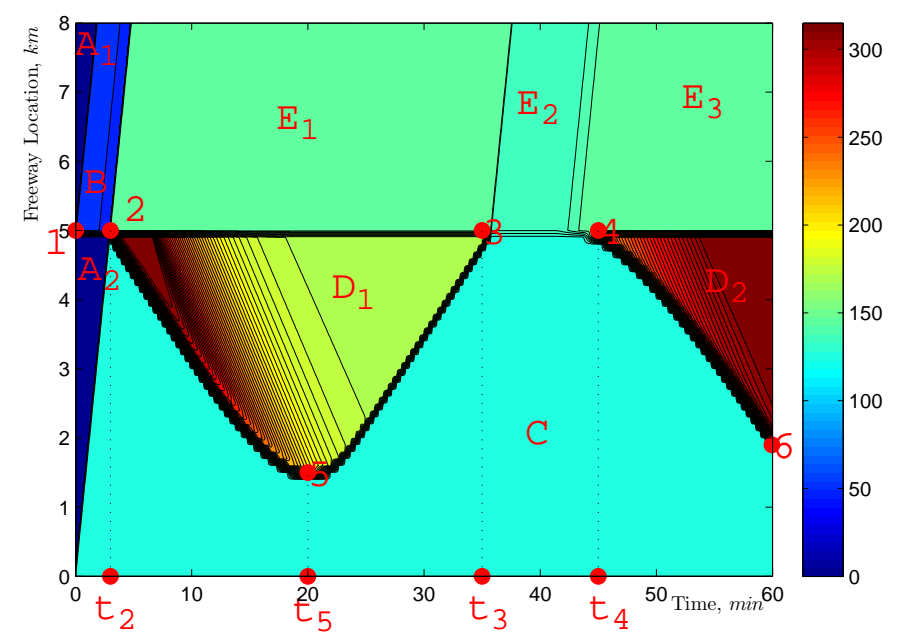

(c) Profile $\alpha_{3}$

Figure 5: Density contours for three different on-ramp inflow profiles in Figure 4 
the hypercongestion gradually disappeared as the inflow rates from the on-ramp continued to fall over time.

In Figure 5(c), point "2" and "4" respectively mark the beginning of each of the two hypercongested regions over the time horizon, corresponding to the two high-demand ends of the entry flow profiles. At first, although the inflow from the on-ramp was decreasing, the inflow was high enough so that, once it was topped on the flow from the freeway upstream, the total demand just was higher than the available capacity at the merge. Then, hypercongestion immediately occurred and propagated upstream, from $t=t_{2}$ up to $t=t_{5}$. As the inflow rate continued to fall, the hypercongested region gradually shrank and eventually disappeared at time $t=t_{3}$. This hypercongestion corresponds to region $\mathbf{D}_{1}$. Clearly, at point "5" the first hypercongested region reached its farthest location upstream and then started to shrink. While the inflow rates stayed flat, the traffic started to settle at a stable state. But at $t=t_{4}$ the inflow rate started to increase again and a new hypercongested region started to form just behind the merge, which corresponds to the hypercongested region $\mathbf{D}_{2}$. Certainly, curves $\mathbf{2}-\mathbf{5}-\mathbf{3}$ and $\mathbf{4}-\mathbf{6}$ respectively correspond to the tails of the two hypercongested regions.

The head position of the hypercongested area remained unchanged and stayed at the merge in Figure 5 (a) when a flat on-ramp inflow profile was applied. However, when the inflow profile was arch-shaped (i.e. one applied in Figure 5(b)), the head position of the hypercongested area in region $\mathrm{D}_{3}$ was moving upwards from the merge. It should be noted that these results were obtained in the setting that the freeway flow from the upstream was constant. When we had a close look at Figure 5(c), it should be reasonable to say that, given the constant freeway flow from the upstrea, the head position of a hyperconegsted area shall stay at the merge, once it is formed, as the on-ramp inflow increases or does not fall and it may move upwards as the inflow falls quickly enough.

\subsubsection{Random on-ramp inflow profile}

Suppose that there exists a random on-ramp inflow profile below:

$$
\alpha^{r}\left(t, x_{\mathrm{on}}\right) \sim\left[0.8 \alpha\left(t, x_{\mathrm{on}}\right), 1.2 \alpha\left(t, x_{\mathrm{on}}\right)\right] \quad \text { referred to as } \alpha_{4}
$$

At each time instant $t$, the inflow $\alpha^{r}\left(t, x_{\mathrm{on}}\right)$ follows a uniform distribution within the interval $\left[0.8 \alpha\left(t, x_{\mathrm{on}}\right), 1.2 \alpha\left(t, x_{\mathrm{on}}\right)\right.$ whose mean is equal to $\alpha\left(t, x_{\mathrm{on}}\right)$; this setting made the resulting density contour map of this profile comparable to the corresponding one presented previously. Figure 6 displays the density contour map corresponding to such a random inflow profile in which $\alpha\left(t, x_{\text {on }}\right)$ was chosen to be the previous valley-shaped inflow profile, i.e. Profile $\alpha_{3}$.

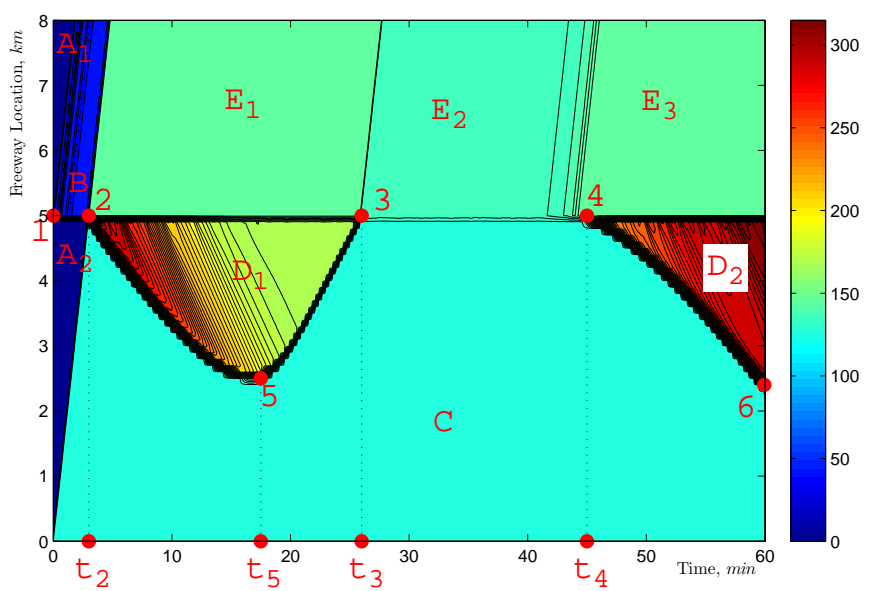

Figure 6: Density contour map from the random on-ramp inflow profile $\alpha_{4}$ in Eq. (24)

As shown in Figure 6, same as the previous deterministic cases, traffic hypercongestion happened just behind the merge. Compare the two density contour maps in Figures 6 and 5(c) and we can see 
that the random profile gave a much smaller hypercongested area than the deterministic one, either in terms of the length of the hypercongested region or the duration the hypercongestion lasted. This point applied to the two hypercongested areas in both density contour maps. In addition, given this random on-ramp inflow profile, the head position of the hypercongested region is clear and was still at the merge but the tail of the region changed randomly although the random change seems invisible in Figure 6; our other numerical experiments (as shown later) showed that the position of the tail fluctuated forward and backward when the inflow randomly changed with a much larger magnitude. Furthermore, the shockwave curves changed their directions randomly in Figure 6 and the overall density in the hypercongested region under the random on-ramp inflow profile looks smaller than that under the associated deterministic profile investigated previously, which helps to explain why the hypercongested region under the random inflow profile was smaller and lasted shorter.

We have also experimented with the random profiles with $\alpha\left(t, x_{\mathrm{on}}\right)$ set to each of the other three deterministic profiles, i.e. $\alpha_{1}, \alpha_{2}$ and $\alpha_{3}$. All results have shown the consistent phenomena as discussed in the previous paragraph.

\subsubsection{A summary}

We have observed the hypercongestion phenomena and investigated the formulation and dissipation of it when different on-ramp inflow profiles were used, both deterministic and stochastic. In addition to what has been said before, it is also worthy pointing out a few more points. First, in all density contour maps regions $\mathbf{A}_{1}$ and $\mathbf{A}_{2}$ correspond to a state that no vehicles were at that location at that time. Second, the traffic in region $\mathbf{B}$ was entirely determined by the inflow from the on-ramp. Third, the change in the sizes of regions $\mathbf{C}$ and $\mathbf{D}$ and their states was dominated by the flow from both the freeway upstream and the on-ramp. Fourth, the upstream traffic streams had some effects on the traffic states in region $\mathbf{E}$ but main impacts on the traffic in this region shall come from the downstream, which is beyond the scope of this paper.

It should be noted that the same or similar clearly recognized regions as those in the density contour maps in Figures 3(a), 5 and 6 are presented and discussed in Laval and Leclercq (2010). But no discussion on traffic hypercongestion was made in this reference while some discussion on on-ramp queues was given. This paper focuses on freeway traffic hypercongestion and does not touch on on-ramp queues.

\subsection{Both on-ramp and off-ramp were operating}

We have investigated traffic hypercongestion on the chosen freeway segment when the upstream off-ramp was closed or the flow leaving from that off-ramp was too little to be worth counting. Now we consider the impacts on traffic hypercongestion of different amounts of traffic leaving from the upstream off-ramp as well as the variation in on-ramp inflow profiles. As specified in Eq. (18), the outflow from the off-ramp was assumed to be proportional to the inflow from the on-ramp (the proportion is denoted by $\theta$ ). As for the on-ramp inflow profiles, we chose three from those experimented previously, i.e. $\alpha_{2}$ (arch-shaped inflow profile), $\alpha_{3}$ (valley-shaped inflow profile) and $\alpha_{4}$ (random profile with $\alpha\left(t, x_{\mathrm{on}}\right)=a$ ). Figure 7 displays the density contour maps for the three chosen profiles with the three specified values of $\theta=0.35,0.65$ and 1. For the convenience of comparison, we should have included those maps corresponding to $\theta=0$ in this figure but that would make each graph too small to be read comfortably.

As pointed out previously, $\theta=0$ may imply that the city and its adjacent area served by the off-ramp of the chosen freeway segment attracted no demand or too little to be worth counting in the time horizon of interest. We can imagine that the attracted demand of the city increased as $\theta$ increased; the increased value of $\theta$ may be associated with the change in the land-use pattern of the city (e.g. gradual transfer from a pure residential place to a residential place but with the rising number of employment positions). In all cases presented in Figure 7, it is assumed that the generated demand from the city remained unchanged. In fact, the absolute demand was not what we were really concerned with but the balance or imbalance between the attracted and generated demand of the city and its adjacent area that used the two ramps of the chosen freeway segment. The balance is captured here by means of the parameter $\theta$. The closer to $1 \theta$ is the more balanced the two types of demand are. $\theta=1$ means that the attracted and 
generated demand of the city and its adjacent area was exactly matched. We did not experiment with $\theta>1$ because traffic hypercongestion would happen as long as $\theta$ was as great as 1 .

Compare the maps in Figure 7 with those in Figures 5(b)-(c) and 6 and then we can gain a number of insights. First, more and more traffic left the freeway via the off-ramp as $\theta$ was increasing and, accordingly, the hypercongested regions gradually become smaller and smaller and eventually disappeared in all three cases of interest, which reduced the duration traffic hypercongestion as well as the average length of the hypercongested region. This means that a more balanced attracted and generated demand of a city and its adjacent area may be able to reduce traffic hypercongestion on those freeways or expressways which pass by or go through the city. Without question, this can also reduce the density on the freeway from the merge downwards.

Second, as $\theta$ rose up the hypercongested density either in the segment between the two ramps or from the diverge upwards continued to fall for each corresponding on-ramp profile, which certainly shortened the journey times of those who did not travel to or from the city served by the two ramps of the chosen freeway segment.

Third, as mentioned before, the constant flow entering from the far upstream of the freeway was $u_{e} \gamma=12,960 \mathrm{veh} / \mathrm{hr}$ and the maximum rate of flow from the on-ramp was $\alpha \delta=5,000 \mathrm{veh} / \mathrm{hr}$; their sum was $17,960 \mathrm{veh} / \mathrm{hr}$, which is above the freeway capacity of $u_{e} \kappa_{e c}=14,400 \mathrm{veh} / \mathrm{hr}$ by $24.72 \%$. If the flow rate leaving the freeway via the off-ramp is less than $5,000-(14,400-12,960)=3,560 \mathrm{veh} / \mathrm{hr}$ (i.e. $71.2 \%$ of the peak amount of the on-ramp inflow) then traffic hypercongestion will be certain to happen behind the merge. However, if the attracted demand of the city and its adjacent area increases whereas the generated demand and the upstream demand of the freeway do not change, we can expect more freeway flow leaving via the off-ramp, which may reduce the probability traffic hypercongestion forms behind the merge.

Fourth, from the third column in Figure 7, we can readily see that no hypercongestion will take place on the chosen homogeneous freeway segment as long as the flow leaving from the upstream off-ramp is not smaller than that entering from the on-ramp. Here, only one simple random on-ramp flow profile was considered. If the freeway flow from the upstream and that from the on-ramp either or both vary randomly with a large magnitude or a high flow level stays for quite a long while, traffic hypercongestion may appear. But as long as these profiles change uniformly and the expected flow leaving via the up-stream off-ramp is not less than the expected downstream on-ramp entry flow, even though hypercongestion may appear, it may not last or become something concerning. Therefore, it would be a good idea to maintain a city to make its attracted and generated time-varying demand profiles can roughly match well.

As can be seen in all cases investigated here, no traffic hypercongestion took place when $\theta=1$. Put all results together and we can draw a reasonable conclusion that no hypercongestion will appear as long as the time-varying profile of outflow via the off-ramp matches well with the profile of inflow from the on-ramp. We did not consider a case in which the on-ramp flow falls while the flow leaving the freeway rises; in fact, from the previous results we can see that no traffic hypercongestion behind the merge will happen in such a situation. All these show that a satellite city and its adjacent area of a metropolitan city shall try to divert or attract as much traffic from the freeway as it can and try offer as many job opportunities as possible so as to reduce or remove traffic hypercongestion between the two ramps serving the satellite city, which can reduce traffic pressure as well as employment pressure the metropolitan city bears.

We did not consider the capacity or entry constraints at the upstream end of the off-ramp in the previous experiments or discussions. If there are such constraints, a queue or hypercongestion will appear when the flow waiting to leave is higher than the available capacity at the approach. The queue or hypercongestion on the off-ramp may also move upwards beyond the diverge so the traffic on the freeway can be affected, which is probably the very thing we should avoid in ramp operations. 


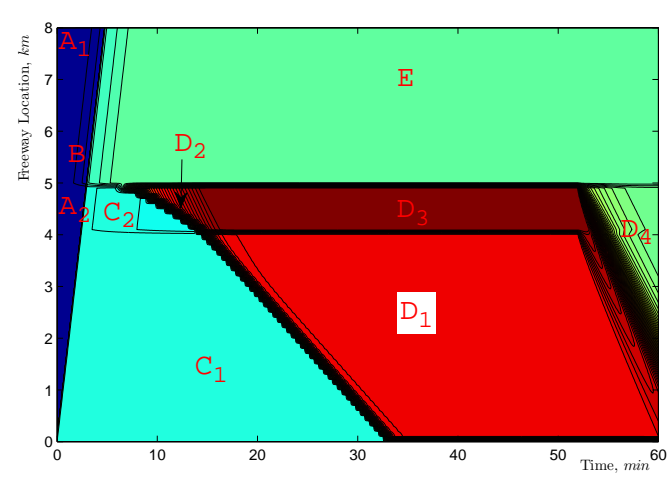

(a) $\alpha_{2} \& \theta=0.35$

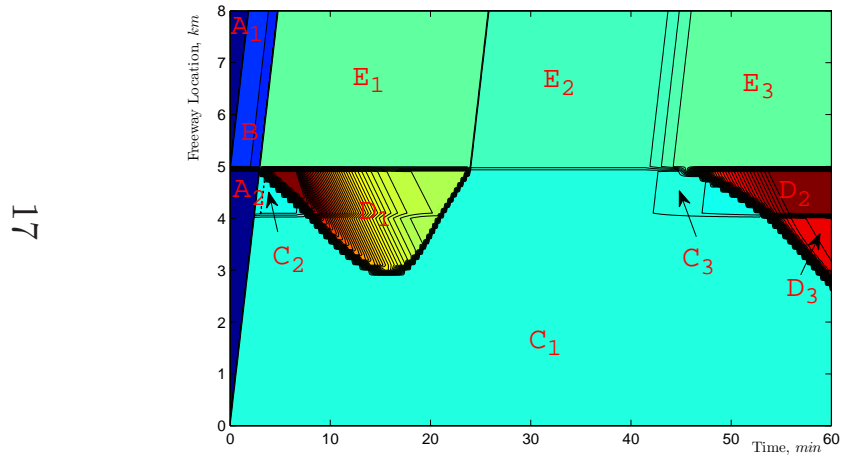

(d) $\alpha_{3} \& \theta=0.35$

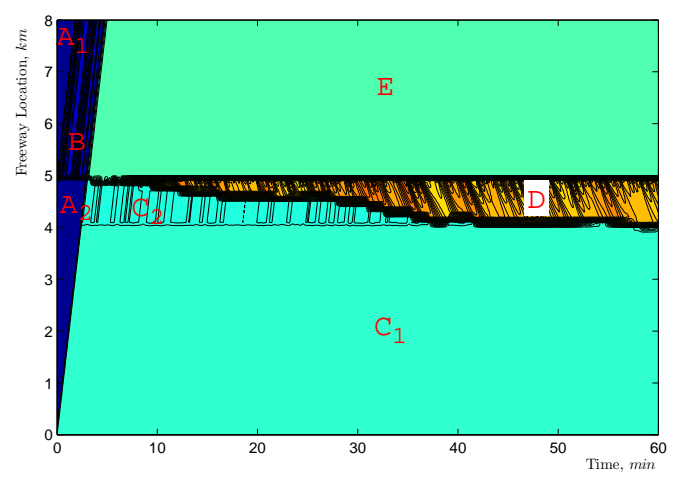

(g) $\alpha_{4} \& \theta=0.35$

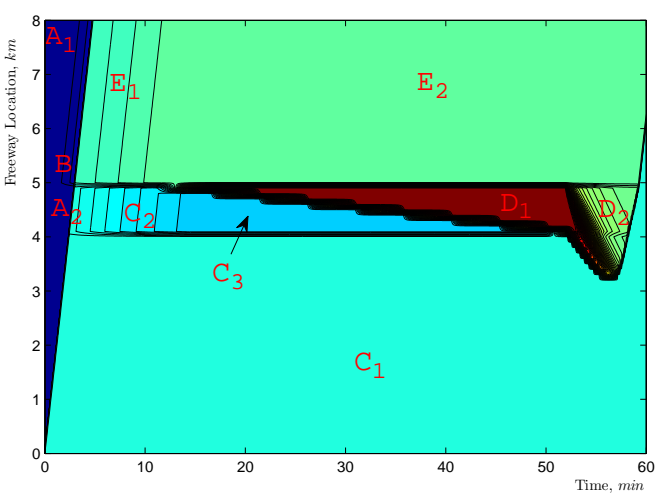

(b) $\alpha_{2} \& \theta=0.65$

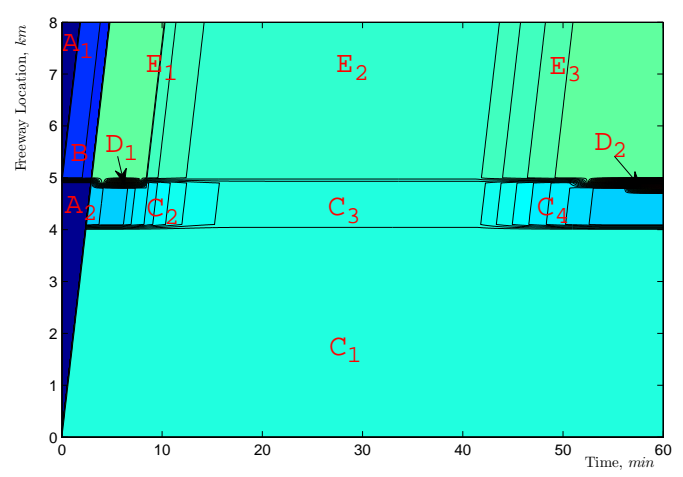

(e) $\alpha_{3} \& \theta=0.65$

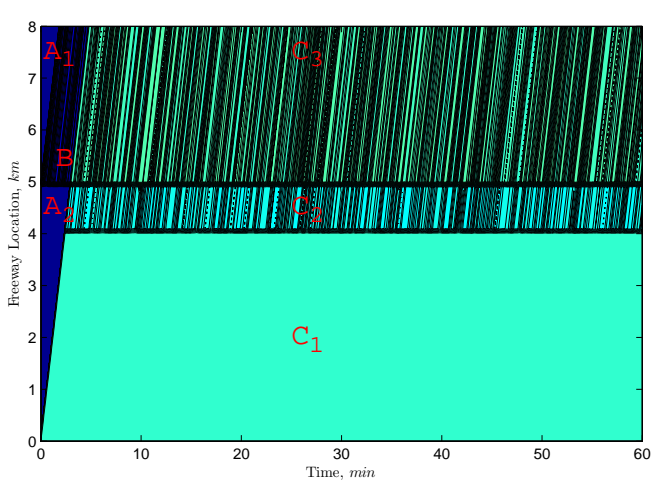

(h) $\alpha_{4} \& \theta=0.65$

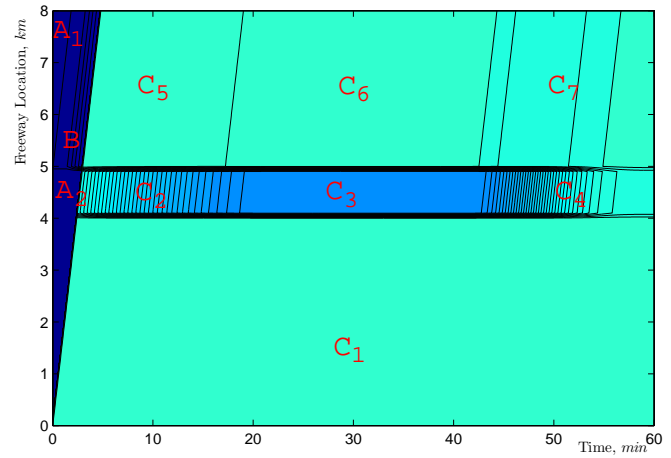

(c) $\alpha_{2} \& \theta=1$

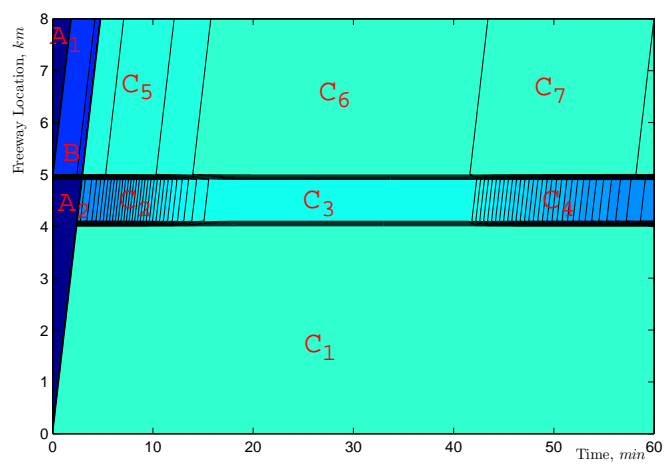

(f) $\alpha_{2} \& \theta=1$

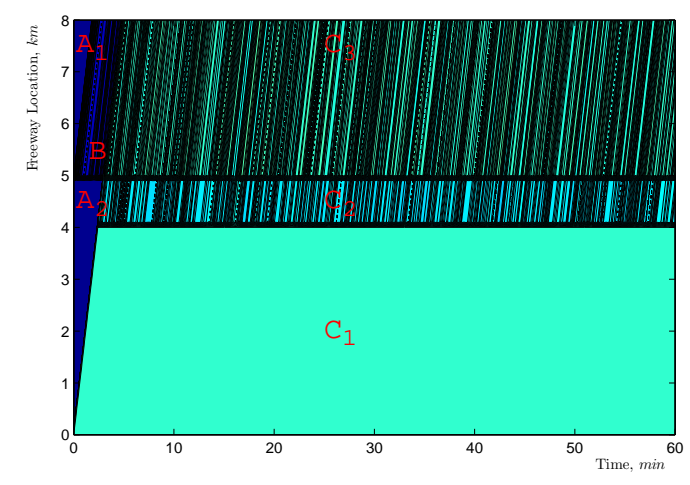

(i) $\alpha_{4} \& \theta=1$

Figure 7: Density contour maps under different on-ramp inflow profiles with varied off-ramp departure proportions 
Our chosen on-ramp inflow profile has a peak lower than the on-ramp capacity. Certainly, the maximum flow can not be greater the capacity and once a flow rate higher the capacity appears a queue will develop at the entry end of the on-ramp. This is out of the scope of this paper.

\section{Concluding remarks}

This paper has used a method modified from the freeway traffic congestion dynamics model in Laval and Leclercq (2010) to investigate traffic hypercongestion on a freeway segment with an on-ramp and an off-ramp situated upstream. We used the distribution scheme satisfying the "fair" condition in Jin and Zhang (2003) to replace the formula for generating the actual rates of inflow from an on-ramp [i.e. Eq. (4)] in Laval and Leclercq (2010). The key reason we made this change is that Eq. (4) in Laval and Leclercq (2010) can not always ensure that the sum of inflow from an on-ramp and the flow on the freeway upstream not greater than the available capacity at the merge. Then we showed numerically that this modified method can produce those phenomena presented in Laval and Leclercq (2010). Then we carried out a series of numerical experiments to analyze the impacts on traffic hypercongestion of varied profiles of on-ramp inflow and off-ramp outflow.

To carry out this investigation, we have chosen a homogeneous freeway segment along which there are an on-ramp and an off-ramp and it is assumed that neither traffic incidents took place nor traffic control facilities existed on these facilities. The characteristics of each facility were assumed to remain unchanged over the time horizon. This scenario may correspond to a typical part of a real-life transportation system where an freeway passes by a city, with an off-ramp lying upstream towards the city and an on-ramp situated downstream. Clearly, the upstream off-ramp and the on-ramp both mainly served the traffic to and from the city.

All numerical experiments can be grouped into two sets. The first set was carried out under the assumption that the upstream off-ramp was closed or the traffic leaving the freeway via the off-ramp was too little to be worth counting. We have seen that a hypercongested region started to formulate once the sum at the merge point of the inflow from the on-ramp plus the flow from the freeway upstream was greater than the available capacity at the merge and it started to shrink and eventually disappeared as the freeway flow or on-ramp flow fell. An interpretation on this case was that the flow on the freeway that was attracted by the city or its adjacent area was zero or too little to be counted since the city was a purely residential place but at the far downstream end of the freeway was a metropolitan city so that a large number of commuters were traveling along the freeway to there to work. Consequently, a severe traffic hypercongestion took place once a high level of flow from the on-ramp started to merge with the flow on the freeway, which lasted long and went upstream easily beyond the off-ramp diverge point.

The second set of numerical experiments investigated the impacts on traffic hypercongestion of varied profiles of on-ramp inflow and off-ramp outflow, with an emphasis on the impacts of the balance between the on-ramp inflow and the off-ramp outflow. Since there was no capacity constraints for either end of the off-ramp, no congestion existed on the off-ramp. Besides, because of the Riemann boundary condition applied to the approach of the off-ramp, no traffic leaving the freeway from the off-ramp was stopped here. Three on-ramp inflow profiles were chosen, i.e. arch-shaped, valley-shaped and one fluctuating in a uniformly-distributed manner. The off-ramp outflow rate at time $t$ was assumed to be proportional to the rate of entry flow of the on-ramp at the same time and the proportion increased from 0 , to 0.35 , to 0.65 and finally to 1 . Our experiments showed that traffic hypercongestion was gradually lessened as the proportion of freeway flow choosing to leave via the off-ramp rose. This can be imagined to be a scenario where a city used to be a purely residential place was gradually converted or grew to be a place with more and more mixed land uses, hence more and more employment positions were available in this city and then higher and higher proportion of the freeway flow was diverted through the off-ramp to this city. This means that the increase in the attracted demand level of the city and its adjacent area can mitigate traffic hypercongestion behind the merge. In other words, under these assumptions given in this paper, these experiments showed that the occurrence or duration of traffic hypercongestion depended on the degree of the balance between attracted and generated demand of the city and its adjacent area the two ramps of the chosen freeway segment served. Without question, a balanced attracted and generated 
demand that were to use the two ramps can reduce the freeway traffic density from a merge downwards, and the journey times who have to experience while traveling down to the far end of the freeway. This shall also be able to reduce traffic pressure in the metropolitan area at the downstream end of the freeway.

This paper assumed no traffic control facilities on the freeway or on the ramps and no capacity constraints on both ends of the off-ramps. In reality, variable/dynamic message signs, ramp metering or other control and management facilities are often installed on freeways to ensure or improve the performance of freeways. An ongoing piece of our work is to minimize the impacts of traffic hypercongestion or remove it by means of, say, ramp operations or control and coordination of flows on freeways and on street roads (e.g. Allen and Newell, 1976; Gugat et al. 2005; Wang et al. 2013; Srivastava and Geroliminis 2013) or improvement in driver behavior in freeway weaving areas (e.g. Sarvi 2013). Another piece of our ongoing work is to put an urban freeway segment into a road network so that we can optimize the whole network, including traffic on the freeway and ramps.

\section{Acknowledgements}

This research is mainly supported by the "Doctoral Fund of Ministry of Education of China" grant (No.: 20110041120009). It is also partly supported by the National Natural Science Foundation of China (Grant No.: 71171026). When the first author was visiting the University of Hong Kong from the middle of December 2012 for three months, he was working on this research and was supported by the Sino-British Fellowship based at this University. The support of all these agencies/organizations is gratefully acknowledged. The authors feel also indebted to the three anonymous referees for their insightful comments on the earlier versions of this paper.

Any opinions, findings, and conclusions or recommendations expressed in this paper are those of the authors and do not necessarily reflect the views of these funding bodies.

\section{References}

[1] Allen, B.L., Newell, G.F. 1976. Some issues relating to metering or closing of freeway ramps. Part I. Control of a single ramp. Part II. Translationally symmetric corridor. Transportation Science 10(3), 227-268.

[2] Bastin, G., B. Haut, J. Coron, B. dAndra Novel. 2007. Lyapunov stability analysis of networks of scalar conservation laws. Networks Heterogeneous Media 2(4), 749757.

[3] Bayen, A., Raffard, R. Tomlin C. 2004. Network congestion alleviation using adjoint hybrid control: Application to highways. R. Alur, G. Pappas, eds. Hybrid Systems: Computation and Control. Springer-Verlag, Berlin, 95110.

[4] Carey, M. and Ge, Y.E. 2005. Convergence of a whole-link travel time model. Transportation Science 39, $25-38$.

[5] Carey, M. and Ge, Y.E. 2012. Comparison of methods for path inflow reassignment for dynamic user equilibrium. Networks and Spatial Economics 12(3): 337-376.

[6] Cassidy, M. and Ahn, S., 2005. Driver turn-taking behavior in congested freeway merges. Transportation Research Record: Journal of the Transportation Research Board, 1934, 140-147.

[7] Coclite, G., M. Garavello, B. Piccoli. 2005. Traffic flow on a road network. SIAM J. Math. Anal. 36(6), 18621886.

[8] Courant, R. and Friedrichs, K. and Lewy, H., 1928. Ber die partiellen differenzengleichungen der mathematischen physik. Mathematische Annalen, 100, 32-74.

[9] Daganzo, C.F., 1994. The cell-transmission model: a simple dynamic representation of highway traffic consistent with the hydrodynamic theory. Transportation Research Part B 28, 269-287.

[10] Daganzo, C.F. 1995. The cell transmission model part II: network traffic. Transportation Research B 29(2), 79-93

[11] Ge, Y.E. and Zhou, X. 2012. An alternative definition of dynamic user optimum on signalized road networks. Journal of Advanced Transportation 46(3): 236-253. 
[12] Godunov, S.K., 1959. A difference method for numerical calculations of discontinuous solutions of the equations of hydrodynamics. Matematicheskii Sbornik, 47, 271-306. (in Russian)

[13] Gugat, M., M. Herty, A. Klar, G. Leugering. 2005. Optimal control for traffic flow networks. J. Optim. Theory Appl. 126(3), 589-616.

[14] Hugoniot, H., 1887. Mémoire sur la propagation des mouvements dans les corps et spécialement dans les gaz parfaits (première partie) [Memoir on the propagation of movements in bodies, especially perfect gases (first part)] (in French) Journal de l'École Polytechnique, 57, 3-97. See also: Hugoniot, H., 1889. Mémoire sur la propagation des mouvements dans les corps et spécialement dans les gaz parfaits (deuxième partie) [Memoir on the propagation of movements in bodies, especially perfect gases (second part)] Journal de l'École Polytechnique, 58, 1-125.

[15] Jin, W.L. and Zhang, H.M., 2003. On the distribution schemes for determining flows through a merge. Transportation Research Part B, 37(6), 512-540.

[16] Laval, J.A. and Leclercq, L., 2008. Microscopic modeling of the relaxation phenomenon using a macroscopic lane-changing model. Transportation Research Part B, 42(6), 511-522.

[17] Laval, J.A. and Leclercq, L., 2010. Continuum approximation for congestion dynamics along freeway corridors. Transportation Science, 87-97.

[18] Lebacque, J.P., 1996. The Godunov scheme and what it means for first order traffic flow models. In: Lesort, J.B.. (Eds.), Proceedings of the 13th International Symposium on Transportation and Traffic Theory, Lyon, France, Publisher: Elsevier, Amsterdam, pp 647-677.

[19] Lebacque J.P. and Khoshyaran 2005. First-Order Macroscopic Traffic Flow Models: Intersection Modeling, Network Modeling. Transportation and Traffic Theory. Flow, Dynamics and Human Interaction. 16th International Symposium on Transportation and Traffic Theory, edited by Hani S Mahmassani, Elsevier, 365-386.

[20] McDonald, J.F., dOuville, E., Liu, L.N. 1999. Economics of Urban Highway Congestion and Pricing, Kluwer.

[21] Newell, G.F. 1993. A simplified theory of kinematic waves in highway traffic: I. General theory; II. Queueing at Freeway Bottlenecks; and III. Multi-Destination Flows. Transportation Research Part B 27(4), 281-313.

[22] Rainer, A., 1990. What does the entropy condition mean in traffic flow theory? Transportation Research Part B, 24(2), 133-143.

[23] Rankine, W.J.M., 1870. On the thermodynamic theory of waves of finite longitudinal disturbances. Philosophical Transactions of the Royal Society of London, 160, 277-288.

[24] Sarvi, M. 2013. Freeway weaving phenomena observed during congested traffic. Transportmetrica A: Transport Science 9(4), 299-315.

[25] Small, K.A., Chu, X. 2003. Hypercongestion. Journal of Transport Economics and Policy 37, 319-352.

[26] Small K.A. and Verhoef E.T. (2007) The Economics of Urban Transportation. Routledge.

[27] Srivastava, A and Geroliminis, N. 2013. Empirical observations of capacity drop in freeway merges with ramp control and integration in a first-order model. Transportation Research Part C 30, 161-177.

[28] Verhoef, E.T. 2001. An integrated dynamic model of road traffic congestion based on simple car-following theory: Exploring hypercongestion. Journal of Urban Economics 49: 505-542.

[29] Wang, YP, E, WJ, Tang, WZ, Tian, DX, Lu, GQ and Yu, gZ 2013. Automated on-ramp merging control algorithm based on Internet-connected vehicles. IET Intelligent Transport Systems 7(4), 371-379. 